\title{
Fatores determinantes da qualidade das águas superficiais na bacia do Alto Acaraú, Ceará, Brasil
}

\author{
Assessment of the surface water quality in the upland of Acaraú watershed, Ceará, Brazil
}

\author{
Eunice Maia de Andrade ${ }^{\mathrm{I}}$ Lúcia de Fátima Pereira Araújo \\ Morsyleide de Freitas Rosa ${ }^{\text {III }}$ Raimundo Benvindo Gomes ${ }^{\text {II }}$ \\ Francisco Antonio de Oliveira Lobato ${ }^{\mathrm{IV}}$
}

\section{RESUMO}

Análise Fatorial/Análise da Componente Principal (AF/ACP) foram empregadas com o objetivo de identificar os fatores determinantes (naturais e antrópicos) da qualidade das águas superficiais na bacia do Alto Acaraú, Ceará. Realizaram-se coletas trimestrais, no período de fev/2003 a mar/2004, em seis pontos localizados nas áreas de influência urbana e de drenagem de perímetros irrigados. Foram analisados os parâmetros $\mathrm{pH}$, temperatura, cor, turbidez, nitrato, amônia total, amônia livre, amônia ionizada, sódio, cloretos, potássio, sulfato, dureza, cálcio, magnésio, alcalinidade total, alcalinidade do bicarbonato, condutividade elétrica, sólidos suspensos, oxigênio dissolvido, demanda bioquímica de oxigênio, fósforo total, ortofosfato solúvel, clorofila "A", coliformes totais e coliformes termotolerantes. Através das técnicas de estatística multivariada, AF/ACP, foram selecionadas as variáveis que explicavam o maior percentual da variância total dos dados. Três componentes são responsáveis pela estrutura da qualidade das águas explicando $88 \%$ da variância total. O modelo mostrou que o primeiro fator (39,81\% da variância) expressou-se como um componente mineral. O segundo, explicando $29,22 \%$ da variância, apresentou-se como um componente de nutrientes. No terceiro fator $(19,16 \%$ da variância), identificou-se como um componente de escoamento superficial das áreas agrícolas e clima. A rotação dos fatores, os parâmetros indicadores da qualidade das águas estão, principalmente, relacionados com a solubilidade dos sais (natural), com nutrientes e transporte de sedimentos (ação antrópica).

Palavras-chave: análise multivariada, monitoramento das águas, poluição hídrica.

\section{ABSTRACT}

Factor analysis/Principal Component Analysis (FA) PCA) were applied to the data set on water quality in upper
Acaraú River basin (Brazil) to study the effects caused by human activities on water. Variables were measured at six key sampling sites (villages and agricultural areas) in four campaigns from February/2003 to March/2004. The samples were analyzed for $\mathrm{pH}$, temperature, color, electrical conductivity, turbidity, nitrate, ammonia, sodium, chloride, potassium, sulfate, total hardness, calcium, magnesium, total alkalinity, alkalinity bicarbonate, total suspended solids, dissolved oxygen (DO), 5-days biochemical oxygen demand (BOD5), total phosphate, orthophosphate, chlorophyll-a, and feacal coliform. Multivariate statistical techniques, (FA/PCA), allowed the identification of variables that explain the major percent of total variance. Three components were identified as responsible for the data structure, explaining $88 \%$ of the total variance of the data set. Model showed that the first factor $39.81 \%$ of variance) assigned as mineralization factor. The second one (29.22\% of variance) represented as nutrients group. The third factor (19.16\% variance) assigned as a combination of agricultural area runoff and weathering. Varimax rotation showed that the mainly water quality parameters were related to mineralization (natural process) and non-point pollution (anthropogenic activities).

Key words: multivariate analysis, water monitoring, water pollution.

\section{INTRODUÇÃO}

A ocupação e o uso do solos decorrentes de atividades humanas alteram sensivelmente os processos biológicos, físicos e químicos dos sistemas naturais. Essas alterações ocorridas em uma bacia hidrográfica podem ser avaliadas através do monitoramento da qualidade das águas superficiais

\footnotetext{
IDepartamento de Engenharia Agrícola, Universidade Federal do Ceará (UFC). Av. Mister Hull S/N, Bloco 804, 60455-970, Fortaleza, CE, Brasil. E-mail: eandrade@ufc.com.br. Autor para correspondência.

${ }^{\text {II } C e n t r o ~ F e d e r a l ~ d e ~ E d u c a c ̧ a ̃ o ~ T e c n o l o ́ g i c a ~ d o ~ C e a r a ́ ~(C E F E T-C E), ~ F o r t a l e z a, ~ C E, ~ B r a s i l . ~}$

IIIEmbrapa Agroindústria Tropical, (EMBRAPA/CNPAT), Fortaleza, CE, Brasil.

${ }^{\mathrm{IV}}$ Curso de Agronomia, UFC, Fortaleza, CE, Brasil.
} 
(MONTEIRO \& PINHEIRO, 2004; SOUZA et al., 2000), uma vez que os rios recebem as descargas industriais, municipais e as águas de drenagem oriundas das áreas exploradas pela agropecuária (PALÁCIO, 2004; SINGH et al., 2005; ELMI et al., 2004). A qualidade da água de uma região é determinada por processos naturais (intensidade das precipitações, intemperismo, cobertura vegetal) e pela influência antrópica (agricultura, concentração urbana, atividade industrial e uso excessivo da água). A exploração incorreta dos solos pela agropecuária representa uma fonte de poluição difusa constante ao longo de todo o ano; enquanto que o escoamento superficial é um fenômeno sazonal, extremamente afetado pelas condições climáticas da bacia (VEGA et al., 1998).

Em regiões áridas e semi-áridas de baixas latitudes, onde não existe o degelo, as barragens e a perenização artificial de rios constituem a principal fonte de água para uso doméstico, industrial e agrícola. A prevenção e o controle da poluição desses mananciais tornam-se imperativos. Através disso, obtêm-se uma real informação da qualidade das águas e a definição do manejo a ser aplicado na bacia. De fato, para se avaliar o estado da qualidade das águas superficiais, é necessário que programas de monitoramento das características físicas, químicas e biológicas sejam implementados, quer em uma visão de variabilidade espacial ou temporal (BRODNJAK-VONCINA et al., 2002; SINGH et al., 2005).

O resultado desses programas é uma enorme e complexa matriz composta por um exaustivo número de informações, quase sempre de difícil interpretação. Por outro lado, a necessidade do controle das fontes de poluição das águas e a definição do manejo a ser aplicado requerem a identificação das fontes poluentes e suas contribuições qualitativas e quantitativas. Para minimizar ou resolver esse problema, técnicas de estatística multivariada como Análise Fatorial/Análise da Componente Principal vêm sendo aplicadas em diferentes partes do globo (FILIZOLA et al., 2002; PALÁCIO, 2004; SILVA \& SACOMANI, 2001; HELENA et al.,2000). Neste estudo, os dados obtidos nas campanhas de coleta foram submetidos à análise estatística multivariada com o objetivo de identificar os fatores determinantes (naturais e antrópicos) da qualidade das águas superficiais da bacia hidrográfica do Alto Acaraú.

\section{MATERIAL E MÉTODOS}

Esta pesquisa foi desenvolvida na bacia hidrográfica do Alto Acaraú (Figura 1), ao norte do Estado do Ceará. Essa bacia detém uma área de $10.000 \mathrm{~km}^{2}$ entre as coordenadas de $3^{\circ} 45^{\prime}$ e $5^{\circ} \mathrm{S}$ e $39^{\circ} 30^{\prime}$ e $41^{\circ} \mathrm{W}$, abrangendo 15 municípios com uma população de 314.455 habitantes. A bacia do Acaraú é drenada pelo rio de mesmo nome, que nasce na Serra da Mata, em cotas superiores a $800 \mathrm{~m}$, desenvolvendo-se no sentido sul-norte. A capacidade total de acumulação da bacia é de aproximadamente $1.215 .390 .000 \mathrm{~m}^{3}$, tendo como principais açudes: o Araras Norte, no rio Acaraú, no município de Varjota, com capacidade de 860,96 milhões de $\mathrm{m}^{3}$; o Edson Queiroz, localizado no rio Groaíras, município de Santa Quitéria, com capacidade de 250 milhões de $\mathrm{m}^{3}$; e o Forquilhas, no rio Madeira, município de Sobral, com capacidade de 50,13 milhões de $\mathrm{m}^{3}$ (DNOCS, 1977b). Esses reservatórios são

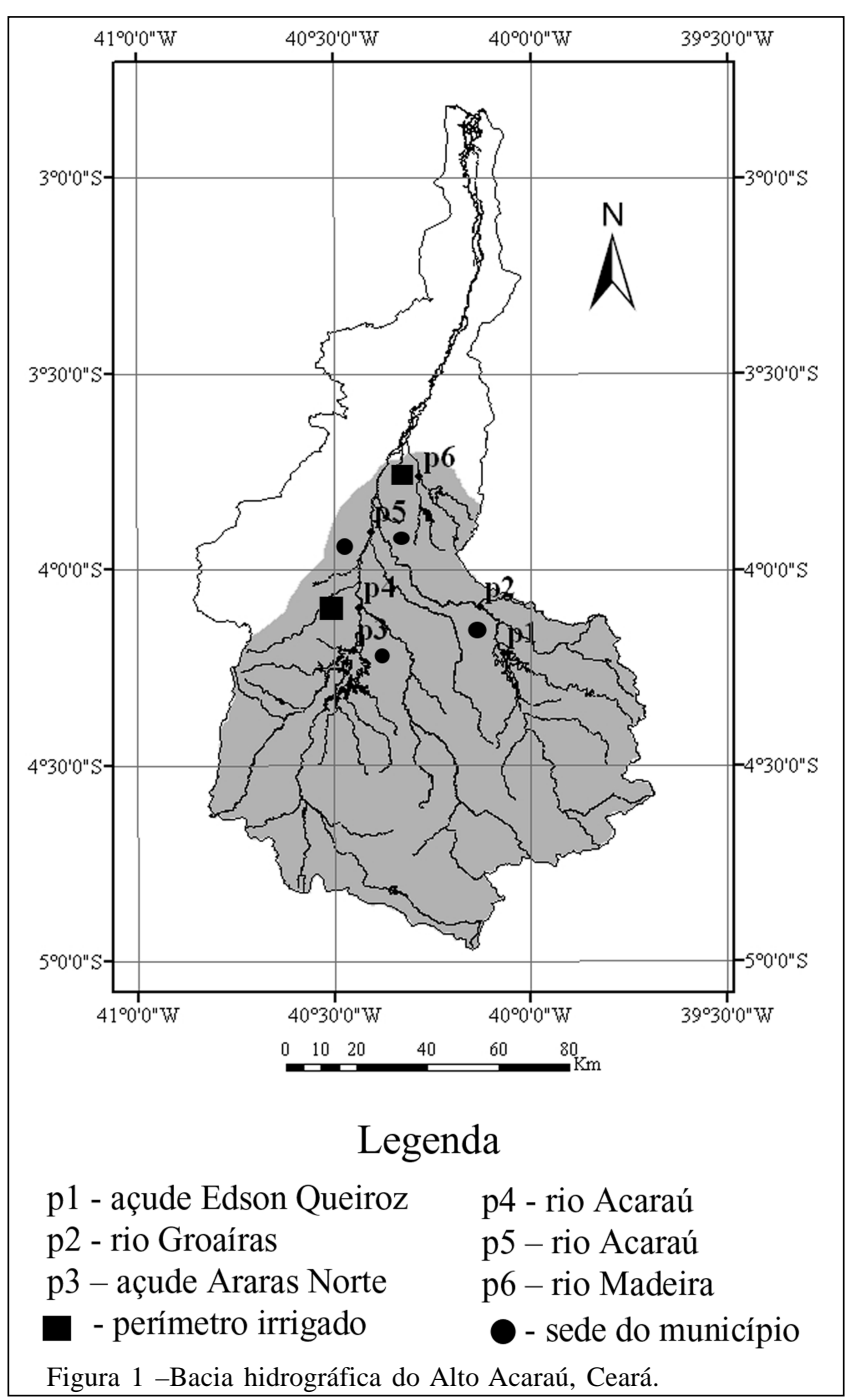

Ciência Rural, v.37, n.6, nov-dez, 2007. 
mananciais de abastecimento humano e de dois perímetros públicos de irrigação.

O clima da região é do tipo BSw'h' - semiárido quente com precipitações de outono e temperaturas médias mensais sempre superiores a $18^{\circ} \mathrm{C}$. A região se caracteriza pela definição de duas estações distintas: uma estação chuvosa, com precipitações irregulares, e outra seca. A pluviosidade média anual da bacia é de aproximadamente $938 \mathrm{~mm}$, com estação chuvosa ocorrendo geralmente de janeiro/fevereiro a maio/junho, concentrando-se $80 \%$ nos meses de março e abril. A evapotranspiração média anual, estimada por tanque classe "A", é de $1.900 \mathrm{~mm}$ e a umidade relativa do ar média situa-se em torno de $70 \%$. Predominam na bacia solos como Luvissolos e Neossolos (CEARÁ, 1992).

Para avaliar o impacto de atividades antrópicas e dos fatores naturais sobre a qualidade da água na bacia do Alto Acaraú, foram realizadas, durante o período fev/2003 a mar/2004, coletas trimestrais de amostras de água, em seis pontos (Figura 1) localizados em áreas de influência de maior densidade urbana e de drenagem de perímetros irrigados. As atividades predominantes na região são: agropecuária, agricultura irrigada e aglomerados populacionais, em sua maioria inferiores a 15.000 habitantes. Foram analisados os parâmetros $\mathrm{pH}$, temperatura, cor, turbidez, nitrato, amônia total, amônia livre, amônia ionizada, sódio, cloretos, potássio, sulfato, dureza, cálcio, magnésio, alcalinidade total, alcalinidade do bicarbonato, condutividade elétrica, sólidos suspensos, oxigênio dissolvido (OD), demanda bioquímica de oxigênio (DBO5), fósforo total, ortofosfato solúvel, clorofila “A”, coliformes totais e coliformes termotolerantes. As análises das amostras de águas seguiram a metodologia apresentada por APHA (1998). A associação entre as variáveis que influenciam a qualidade das águas superficiais foi identificada mediante as técnicas de estatística multivariada, Análise Fatorial/Análise do Componente Principal (AF/ACP). Para a análise dos dados, foi utilizado o SPSS (Statistical Package for the Social Sciences), por apresentar bastante versatilidade no manuseio das operações necessárias à obtenção de componentes principais, contando inclusive com o tratamento prévio de padronização e escalonamento dos dados.

Os dados originais, na forma de matriz, eram expressos por $\mathrm{X}=\left(\mathrm{x}_{\mathrm{i}, \mathrm{j}}\right)$, em que $\mathrm{i}=1$...n amostragens (24) e j = 1...p variáveis (16). Na aplicação da técnica da Análise Fatorial/Análise do Componente Principal (AF/ $\mathrm{ACP}$ ), a primeira etapa é transformar a matriz de dados originais em uma matriz de correlação [R] (p x p), para p igual aos 16 parâmetros de qualidade de água analisados neste estudo. A principal razão para se usar a matriz de correlação é se eliminar o problema de escalas e unidades diferenciadas em que as variáveis são medidas. Outras informações sobre o assunto podem ser encontradas em DILLON \& GOLDSTEIN (1984) e JOHNSON \& WICHERN (1978). A eficiência da nova descrição dos dados através das componentes vai depender da percentagem de variação total que cada componente contém, a qual é representada pelos autovalores (MOITA NETO, 1997; VEGA et al., 1998).

Após a definição da matriz de correlação, realizou-se uma inspeção entre os parâmetros com o objetivo de identificar as variáveis mais específicas, visto que a finalidade da ACP é obter componentes que ajudem a explicar essas correlações. A consistência geral dos dados foi aferida pelo método Kayser Mayer Olkim (KMO), que compara a magnitude dos coeficientes de correlação observados com os coeficientes de correlação parcial, produzindo um índice KMO (MONTEIRO \& PINHEIRO, 2004; ANDRADE et al., 2003). A seleção do número de componentes extraído seguiu os preceitos apresentados por NORUSIS (1990), em que se consideram somente componentes com variância superior a um. Este critério fundamenta-se no fato de que qualquer fator deve explicar uma variância superior àquela apresentada por uma simples variável.

O primeiro componente principal extraído foi a combinação linear com variância máxima existente na amostra; a segunda, a combinação linear com a máxima variância remanescente; e assim sucessivamente. Componentes sucessivas explicam porções progressivamente menores da variância da amostra total, sem apresentar correlação com as componentes anteriores. Segundo NORUSIS (1990), o modelo matemático para análise de fator apresenta semelhança com uma equação de regressão múltipla, sendo cada variável expressa como uma combinação linear de fatores que não são observados de fato. O modelo para a i-ésima variável normalizada é escrito através da regressão linear múltipla entre fatores:

$X s i=A_{i 1} F_{1}+A_{i 2} F_{2}+\ldots+A_{i l} F_{k_{i}} \ldots+A_{i L} F_{L}+\xi$

sendo F: fatores comuns, isto é, uma nova variável; Ai: constantes de ajuste do modelo $(i=1, \ldots \mathrm{L})$; $\xi$ : erro experimental; $\mathrm{L}$ : total de fatores.

O modelo assume que os erros experimentais não têm correlação com os fatores comuns (PALÁCIO, 2004). Os fatores são deduzidos das variáveis observadas e podem ser calculados como combinações lineares dessas variáveis. É possível que todas as variáveis contribuam para o fator de qualidade da água; no entanto, espera-se que um único subconjunto de 
variáveis caracterize a qualidade da água, indicado pelos maiores coeficientes. A expressão geral para a estimativa do k-ésimo fator $F_{k}$ é:

$\mathrm{f}_{\mathrm{k}}=\sum_{\mathrm{i}=1}^{\mathrm{p}} \mathrm{W}_{\mathrm{ki}} \mathrm{X}_{\mathrm{i}}=\mathrm{W}_{\mathrm{k} 1} \mathrm{X}_{1}+\mathrm{W}_{\mathrm{k} 2} \mathrm{X}_{2}+\ldots+\mathrm{W}_{\mathrm{kp}} \mathrm{X}_{\mathrm{p}}$ em que $\mathrm{W} k \mathrm{ki}$ : peso da i-ésima variável no k-ésimo fator; Xi: escore atribuído a cada variável; p: número de variáveis. Mesmo com a matriz dos componentes obtidos na fase de extração, na qual o resultado descreve a relação entre os fatores e as variáveis individuais, o resultado pode ser de difícil interpretação. Para suplantar a limitação, utilizou-se o procedimento de transformação ortogonal, ou simplesmente rotação da matriz das cargas fatoriais, gerando uma nova matriz de cargas fatoriais, que apresenta um melhor significado interpretativo aos fatores. Neste estudo, empregou-se a rotação ortogonal, método Varimax, empregando-se a avaliação da variabilidade espacial e temporal da qualidade de água (PALÁCIO, 2004; VEGAet al., 1998).

\section{RESULTADOS E DISCUSSÃO}

Embora tenham sido analisadas 26 variáveis, o modelo da AF/ACP mostrou-se adequado para uma matriz de correlação composta por 16 delas, uma vez que as 10 restantes não se mostraram significativas na explicação da variância total. A matriz de correlação composta pelas variáveis empregadas neste trabalho pode ser vista nas tabelas 1 e 2 . Pela referida matriz, observa-se que todas as variáveis estudadas apresentaram correlação superior a 0,5 com pelo menos três outras variáveis. HELENA et al. (2000) consideram que coeficiente de correlação superior a 0,5 expressa uma forte relação. Pode-se, também, observar que CE,
$\mathrm{Na}^{+}, \mathrm{Ca}^{+2}, \mathrm{Mg}^{+2}$, dureza e alcalinidade do bicarbonato apresentaram correlações superiores a 0,9.

As altas correlações registradas entre sódio, dureza, cálcio, magnésio e alcalinidade do bicarbonato podem ser explicadas pela presença de rochas hornblendas na geologia da parte alta da bacia do Acaraú (DNOCS, 1977a). A correlação muito forte da condutividade elétrica com o sódio, o magnésio, o cálcio, a dureza e o cloreto era esperada, uma vez que a condutividade elétrica expressa a concentração dos sais na água. Resultados semelhantes foram encontrados por PALÁCIO (2004), ao estudar a variação temporal da composição das águas do aqüífero aluvial do rio Trussu, na parte alta da bacia do Jaguaribe, Ceará. Já o elevado teor de nitrato, sulfato e fósforo nas águas não pode ser atribuído à geologia da bacia, uma vez que os mesmos não fazem parte da formação geológica local (DNOCS, 1977a). Acredita-se que as concentrações desses elementos sejam decorrentes das precárias condições de infra-estrutura de saneamento básico dos aglomerados urbanos e de atividades agropecuárias.

O teste de adequacidade aplicado ao modelo, Kaiser-Meyer-Olkin (KMO), apresentou um índice igual a 0,625 (regular), demonstrando que o modelo promoverá significante redução na dimensão dos dados originais. Pelo teste da Análise Fatorial/ Análise do Componente Principal (AF/ACP), foi selecionado um modelo com três componentes. A seleção do número de componentes teve como base os princípios descritos por NORUSIS (1990), ou seja, considerar somente aqueles componentes que apresentem um autovalor superior a um.

Tabela 1 - Matriz de correlação das variáveis indicadoras da qualidade das águas na bacia do Alto Acaraú, Ceará.

\begin{tabular}{|c|c|c|c|c|c|c|c|c|}
\hline & $\mathrm{pH}$ & Cor & Turb & $\mathrm{NO}_{3}$ & $\mathrm{Na}^{2+}$ & $\mathrm{K}^{+}$ & $\mathrm{SO}_{4}$ & Dur \\
\hline $\mathrm{pH}$ & 1,00 & & & & & & & \\
\hline Cor & $\underline{-0,63}$ & 1,00 & & & & & & \\
\hline Turb & $-0,66$ & $\underline{0,97}$ & 1,00 & & & & & \\
\hline $\mathrm{NO}_{3}$ & 0,47 & 0,14 & 0,14 & 1,00 & & & & \\
\hline $\mathrm{Na}^{2+}$ & 0,02 & 0,02 & 0,12 & 0,17 & 1,00 & & & \\
\hline $\mathrm{K}^{+}$ & $\underline{0,60}$ & $-0,16$ & $-0,16$ & $\underline{0,80}$ & 0,33 & 1,00 & & \\
\hline $\mathrm{SO}_{4}$ & 0,44 & 0,16 & 0,16 & $\underline{0,90}$ & 0,17 & $\underline{0,79}$ & 1,00 & \\
\hline Dur & $-0,05$ & 0,02 & 0,13 & 0,07 & $\underline{0,95}$ & 0,23 & 0,004 & 1,00 \\
\hline $\mathrm{Ca}^{2+}$ & $-0,00$ & 0,01 & 0,11 & 0,13 & $\underline{0,90}$ & 0,34 & 0,03 & $\underline{0,96}$ \\
\hline $\mathrm{Mg}^{2+}$ & $-0,07$ & 0,03 & 0,14 & 0,02 & $\underline{0,95}$ & 0,15 & $-0,01$ & $\underline{0,98}$ \\
\hline$A B$ & 0,02 & $-0,02$ & 0,08 & 0,13 & $\underline{0,96}$ & 0,26 & 0,07 & $\underline{0,97}$ \\
\hline CE & 0,12 & $-0,01$ & 0,08 & 0,27 & $\underline{0,98}$ & 0,44 & 0,27 & $\underline{0,93}$ \\
\hline SST & $\underline{-0,74}$ & $\underline{0,56}$ & $\underline{0,51}$ & $-0,42$ & $-0,03$ & $\underline{-0,54}$ & $-0,47$ & 0,06 \\
\hline $\mathrm{P}$ & 0,40 & 0,09 & 0,12 & $\underline{0,60}$ & $\underline{0,52}$ & $\underline{0,56}$ & $\underline{0,72}$ & 0,33 \\
\hline OS & 0,37 & 0,16 & 0,18 & $\underline{0,57}$ & $\underline{0,54}$ & $\underline{0,54}$ & $\underline{0,69}$ & 0,36 \\
\hline $\mathrm{Cl}$ & 0,22 & 0,10 & 0,15 & 0,28 & $\underline{0,73}$ & 0,49 & 0,41 & $\underline{0,64}$ \\
\hline
\end{tabular}

Ciência Rural, v.37, n.6, nov-dez, 2007. 
Tabela 2 - Matriz de correlação das variáveis indicadoras da qualidade das águas na bacia do Alto Acaraú, Ceará.

\begin{tabular}{|c|c|c|c|c|c|c|c|c|}
\hline & $\mathrm{Ca}^{2+}$ & $\mathrm{Mg}^{2+}$ & $\mathrm{AB}$ & $\mathrm{CE}$ & SST & $\mathrm{P}$ & OS & $\mathrm{Cl}$ \\
\hline $\mathrm{Ca}^{2+}$ & 1,00 & & & & & & & \\
\hline $\mathrm{Mg}^{2+}$ & $\underline{0,89}$ & 1,00 & & & & & & \\
\hline$A B$ & $\underline{0,93}$ & $\underline{0,95}$ & 1,00 & & & & & \\
\hline CE & $\underline{0,89}$ & $\underline{0,92}$ & $\underline{0,95}$ & 1,00 & & & & \\
\hline SST & 0,01 & 0,08 & $-0,03$ & $-0,12$ & 1,00 & & & \\
\hline $\mathrm{P}$ & 0,28 & 0,35 & 0,39 & $\underline{0,57}$ & $-0,35$ & 1,00 & & \\
\hline OS & 0,29 & 0,39 & 0,40 & $\underline{0,60}$ & $-0,36$ & $\underline{0,94}$ & 1,00 & \\
\hline $\mathrm{Cl}$ & $\underline{0,57}$ & $\underline{0,67}$ & $\underline{0,62}$ & $\underline{0,77}$ & $-0,24$ & $\underline{0,68}$ & $\underline{0,79}$ & 1,00 \\
\hline
\end{tabular}

Cor (Pt/Co); Turb (FTU) - Turbidez; $\mathrm{NO}_{3}\left(\mathrm{mgN} \mathrm{L}^{-1}\right)$ - nitrato; $\mathrm{Na}\left(\mathrm{mg} \mathrm{L}^{-1}\right)$ - Sódio; K(mg L $\left.{ }^{-1}\right)$ - Potássio; $\mathrm{SO}_{4}\left(\mathrm{mgN} \mathrm{L}^{-1}\right)$ - Sulfato; Dur - Dureza (mg $\left.\mathrm{L}^{-1}\right)$; Ca (mg L $\left.{ }^{-1}\right)$ - Cálcio; $\mathrm{Mg}\left(\mathrm{mg} \mathrm{L}^{-1}\right)$ - Magnésio; $\mathrm{AB}$ - Alcalinidade do Bicarbonato $\left(\mathrm{mgCaCO}_{3} \mathrm{~L}^{-1}\right)$; $\mathrm{CE}\left(\mathrm{dS} \mathrm{m}^{-1}\right)$ - Condutividade elétrica; SST $\left(\mathrm{mg} \mathrm{L}^{-1}\right)$ - Sólidos solúveis totais; $\mathrm{P}\left(\mathrm{mgP} \mathrm{L}^{-1}\right)$ - Fósforo total; OS $\left(\mathrm{mgP} \mathrm{L}^{-1}\right)$ Ortofosfato Solúvel; $\mathrm{Cl}\left(\mathrm{mgCl} \mathrm{L}^{-1}\right)-\mathrm{Cloreto}$.

Os pesos fatoriais atribuídos a cada componente, bem como a percentagem da variância total explicada por cada componente, podem ser vistas na tabela 3. Verifica-se que os três primeiros componentes explicaram respectivamente 46,44, e 25,12 e 16,62\% da variância total dos dados, concentrando em três dimensões 88,18\% das informações antes dissolvidas em 16 dimensões. Estes resultados confirmam aqueles encontrados por PALÁCIO (2004), trabalhando com águas de rio Trussu, no Ceará, onde encontrou um modelo formado por dois componentes, explicando 83,43\% da variância total.

Pela tabela supracitada podem ser observados os valores dos pesos fatoriais para as

Tabela 3 - Matriz do peso fatorial das variáveis nos três componentes principais selecionados.

\begin{tabular}{llll}
\hline Variáveis & CP 1 & CP 2 & CP 3 \\
\hline Condutividade Elétrica (CE) & $\underline{\underline{0,964}}$ & 0,206 & $-0,124$ \\
Sódio & $\underline{\underline{0,929}}$ & 0,320 & $-0,131$ \\
Alcalinidade do bicarbonato & $\underline{\underline{0,874}}$ & 0,374 & $-0,227$ \\
Dureza & $\underline{0,853}$ & 0,461 & $-0,209$ \\
Cloretos & $\underline{0,844}$ & $-0,038$ & 0,101 \\
Magnésio & $\underline{0,836}$ & 0,483 & $-0,192$ \\
Cálcio & $\underline{0,825}$ & 0,399 & $-0,221$ \\
Ortofosfato solúvel & $\underline{0,751}$ & $-0,358$ & 0,341 \\
Fósforo total & $\underline{0,727}$ & $-0,407$ & 0,309 \\
pH & 0,267 & $\underline{-0,802}$ & $-0,399$ \\
Sólidos suspensos & $-0,260$ & $\underline{0,757}$ & 0,327 \\
Sulfato & 0,479 & $\underline{-0,681}$ & 0,484 \\
Potássio & 0,599 & $\underline{-0,633}$ & 0,062 \\
Nitrato & 0,471 & $\underline{-0,628}$ & 0,416 \\
Cor & 0,026 & 0,401 & $\underline{0,897}$ \\
Turbidez & 0,112 & 0,446 & $\underline{0,861}$ \\
Autovalor & 7,43 & 4,02 & 2,65 \\
\% variância explicada & 46,44 & 25,12 & 16,62 \\
\% variância acumulada & 46,44 & 71,56 & 88,18 \\
\hline
\end{tabular}

CP - Componente Principal. componentes um, dois e três (CP 1; CP 2 e CP 3). Esses componentes expressam a relação entre fatores e variáveis e permitem a identificação das variáveis com maiores inter-relações em cada componente. Os valores elevados dos pesos fatoriais sugerem quais são as variáveis mais significativas em cada fator. No primeiro fator, as variáveis CE, sódio, alcalinidade do bicarbonato, dureza, cloreto, cálcio e magnésio apresentaram um peso superior a 0,82, indicando que estas variáveis são as mais significativas na definição da qualidade da água estudada. Portanto, fica evidente que a CP 1 está relacionada com o processo natural de intemperismo dos componentes geológicos do solo (BRODNJAK-VONCINA et al., 2002). Já a segunda CP é explicada, principalmente, pelas variáveis $\mathrm{pH}$, sólidos suspensos, sulfato, potássio e nitrato (peso $>0,62$ ), as quais indicam o uso da terra pela agropecuária. A presença do nitrato em águas superficiais pode ter sua origem em fertilizantes orgânicos, enquanto que o sulfato e o potássio em fertilizantes químicos (ELMI et al., 2004). A CP 3 apresentou uma maior interrelação com cor e turbidez (peso >0,8), sugerindo ser estas as únicas variáveis significativas neste componente. Este componente, basicamente, expressa o efeito do escoamento superficial com uma carga de sedimentos oriundos das áreas agrícolas e a contribuição de esgotos e resíduos sólidos dispostos, inadequadamente, próximos às margens dos cursos d'águas. Segundo dados do Censo 2000 (IBGE,2002), 40\% dos domicílios da área urbana e 99\% da área rural realizam a queima, enterram ou dispõem o lixo em terreno baldio, rio, lago, mar ou então destino que não a coleta. A influência da erosão hídrica, também, foi identificada por SINGH et al. (2005) em estudos de qualidade de água e aportes de poluição em águas do Gomti, na Índia. O modelo aplicado mostrou que, para o primeiro componente, foram significantes os parâmetros relacionados com os sais dissolvidos na água; 
enquanto que, para o segundo, os elementos relacionados com a poluição agrícola apresentaram maior significância e, para o terceiro, os maiores pesos foram atribuídos ao escoamento superficial.

Em geral, a matriz do peso fatorial (Tabela 3) apresenta dificuldades na identificação das variáveis mais significativas, em decorrência de valores muito próximos entre si (DILLON \& GOLDSTEIN, 1984). Para suplantar essa limitação, aplicou-se a transformação ortogonal pelo emprego do algoritmo varimax. Pesquisadores como ANDRADE et al.(2003), PALÁCIO (2004), SILVA \& SACOMANI (2001) obtiveram uma matriz de mais fácil interpretação com a aplicação do algoritmo varimax na elaboração da matriz transformada. A adoção da matriz transformada, neste estudo, gerou mudanças significativas em relação à matriz original (Tabela 4). Pode-se observar uma melhor distribuição da variância total entre as três componentes. Comparando-se as tabelas 3 e 4, verifica-se uma redução do percentual da variância total explicada pela CP 1 e um conseqüente aumento do percentual da variância explicado pela CP 2 e pela CP 3, sem ocorrer variação do total explicado pelo modelo, como afirmavam MONTEIRO \& PINHEIRO (2004). Após a rotação, VF 1 expressou uma maior associação com os parâmetros indicadores do enriquecimento por íons solúveis (pesos > 0,941). Uma menor associação foi registrada com o cloreto, ocorrendo um acréscimo do

Tabela 4 - Fatores dos componentes da matriz transformada pelo algoritmo varimax.

\begin{tabular}{llll}
\hline Variáveis & VF 1 & VF 2 & VF 3 \\
\hline Dureza & $\underline{0,990}$ & 0,030 & 0,045 \\
Magnésio & $\underline{0,982}$ & 0,014 & 0,071 \\
Alcalinidade do bicarbonato & $\underline{\underline{0,973}}$ & 0,094 & $-0,018$ \\
Sódio & $\underline{0,969}$ & 0,207 & 0,031 \\
Condutividade elétrica (CE) & $\underline{0,944}$ & 0,309 & $-0,026$ \\
Cálcio & $\underline{0,941}$ & 0,054 & 0,002 \\
Cloretos & $\underline{0,671}$ & 0,523 & 0,035 \\
Sulfato & $-0,037$ & $\underline{0,963}$ & 0,020 \\
Nitrato & $-0,002$ & $\underline{0,888}$ & $-0,008$ \\
Fósforo total & 0,346 & $\underline{0,819}$ & 0,014 \\
Ortofosfato solúvel & 0,381 & $\underline{0,812}$ & 0,066 \\
Potássio & 0,197 & $\underline{0,792}$ & $-0,312$ \\
Cor & $-0,029$ & 0,159 & $\underline{0,969}$ \\
Turbidez & 0,074 & 0,156 & $\underline{0,961}$ \\
pH & $-0,039$ & 0,514 & $\underline{-0,779}$ \\
Sólidos suspensos & 0,044 & $-0,513$ & $\underline{0,695}$ \\
Autovalor & 6,37 & 4,68 & 3,07 \\
\% variância explicada & 39,81 & 29,22 & 19,16 \\
\% variância acumulada & 39,81 & 69,03 & 88,18 \\
\hline
\end{tabular}

VF - varimax fator. peso deste parâmetro para a VF 2, podendo isso ser um indicativo da origem de sais não naturais, os quais podem ser atribuídos ao aporte de esgotos ao rio ou ao carreamento de fertilizantes químicos. HELENA et al. (2000) associaram a contribuição de esgotos residenciais e industriais a uma maior concentração de cloreto nas águas aluvionais do rio Pisuerga, na Espanha. A VF 2 passou a ser composta por sulfato, nitrato, fósforo total, ortofosfato solúvel e potássio, sendo eliminado o percentual de explicação da variância pelo $\mathrm{pH}$ para esta componente. A VR 2 sugere que as atividades agrícolas e pastoris da região, fontes de poluição difusa, influenciam significativamente a concentração dos nutrientes nas águas superficiais. $\mathrm{O}$ nitrato e o fósforo, elementos definidos pela literatura como indicadores das atividades agropecuárias (BROOKS et al., 1992; PALÁCIO, 2004; RAVICHANDRAN et al., 1996), fazem-se presentes nesse componente com pesos superiores a 0,8. A VF 3 mostrou inter-relação com a cor, a turbidez, os sólidos suspensos e o $\mathrm{pH}$, apresentando-se como um fator de transporte de sedimentos. Os altos pesos $(>0,96)$ atribuídos à cor e à turbidez podem ser explicados pelas características climáticas das regiões semi-áridas (baixa cobertura vegetal e chuvas de alta intensidade).

\section{CONCLUSÕES}

O emprego da Análise Fatorial/ Análise do Componente Principal (AF/ACP) promoveu a redução de 16 características das águas superficiais da bacia do rio Acaraú para três componentes, que explicam $88 \%$ da variância total. A rotação dos fatores mostrou que os parâmetros indicadores da qualidade das águas na bacia do Alto Acaraú estão, principalmente, relacionados com a solubilidade dos sais (ação natural), com nutrientes e transporte de sedimentos (ação antrópica), sendo o principal fator a geologia da região. A poluição difusa, gerada nos núcleos urbanos e em atividades agropecuárias, apresentou-se como o segundo fator determinante da qualidade das águas superficiais na área em estudo.

\section{AGRADECIMENTOS}

016/01-01.

$$
\text { Pesquisa financiada pelo PRODETAB - Projeto }
$$

\section{REFERÊNCIAS}

ANDRADE, E.M. de et al. Investigação da estrutura multivariada da evapotranspiração na região centro sul do Ceará pela análise de componentes principais. Revista Brasileira de Recursos Hídricos, v.8, n.1, p.39-44, 2003. 
APHA. Standard methods for the examination of water and wastewater. 20.ed. Washington, DC: American Public Health Association, 1998. 1220p.

BRODNJAK-VONCINA, D. et al. Chemometrics characterisation of the quality of river water. Analytica Chimica Acta, v.462, p.87-100, 2002.

BROOKS, K.N. et al. Hydrology and the management of watersheds. Ames: Iowa State University, 1992. 392p.

CEARÁ, Secretaria dos Recursos Hídricos. Plano estadual dos recursos hídricos: Estudo de base II. Fortaleza, CE, 1992. V.2. 1471p.

DILLON, W.R.; GOLDSTEIN, M. Multivariate analysis methodos and applications. New York: John Wiley e Sons, 1984. 587p.

DNOCS. Plano diretor do Vale do Acaraú. Pedologia. Fortaleza, 1977a. V.2, 96p.

DNOCS. Plano diretor do Vale do Acaraú. Diagnóstico. Fortaleza, 1977b. V.1, 136p.

ELMI, A.A. et al. Water and fertilizer nitrogen management to minimize nitrate pollution from a cropped soil in southwestern Quebec, Canada. Water, Air, and Soil Pollution, v.151, p.117-134, 2004.

FILIZOLA, H.F. et al. Monitoramento e avaliação do risco de contaminação por pesticidas em águas superficial e subterrânea na região de Guairá. Pesquisa Agropecuária Brasileira, v.37, n.5, p.659-667, 2002.

HELENA, B. et al. Temporal evolution of groundwater composition in an alluvial aquifer (Pisuerga river, Spain) by principal component analysis. Water Research, v.34, n.3, p.807-816, 2000.

IBGE. Indicadores de desenvolvimento sustentável: Brasil 2002. Rio de Janeiro, 2002. 195p.
JOHnSON, R.A.; WICHERN, D.W. Applied regression analysis and other multivariate. New Jersey: Prentice Hall, 1978. 357p.

MOITA NETO, J.M. Uma introdução à análise exploratória de dados multivariados. Química Nova, v.24, n.4, p.467-469, 1997.

MONTEIRO, V.P.; PINHEIRO, J.C.V. Critério para implantação de tecnologias de suprimentos de água potável em municípios cearenses afetados pelo alto teor de sal. Revista de Economia Rural, v.42, n.2, p.365-387, 2004.

NORUSIS, M.J. SPSS Base System user's guide. Chicago: SPSS, 1990. 520p.

PALÁCIO, H.A. de Q. Índice de qualidade das águas na parte baixa da bacia hidrográfica do rio Trussu, Ceará 2004. 96f. Dissertação (Mestrado em Irrigação e Drenagem) - Universidade Federal do Ceará, Fortaleza.

RAVICHANDRAN, S. et al. Ecoregions for describing water quality patterns in Tamiraparani basin, South India. Journal of Hydrology, v.178, p.257-276, 1996.

SILVA, A.M.M.; SACOMANI, L.B. Using chemical and physical parameters to define the quality of Pardo river water (Botucatu- SP-Brasil). Water Research, v.35, n.6, p.16091616, 2001

SINGH, K.P. et al. Water quality assessment and apportionment of pollution sources of Gomti river (India) using multivariate statistical techniques-a case study. Analytica Chimica Acta, v.515, p.143-149, 2005.

SOUZA, L.C. de, et al. Variabilidade espacial da salinidade de um solo aluvial no semi-árido paraibano. Revista Brasileira de Engenharia Agrícola e Ambiental, v.4, n.2, p.35-40, 2000 .

VEGA, M. et al. Assessement of seasonal and polluting effects on the quality of river water by exploratory data analysis. Water Research, v.32, n.12, p.3581-3592, 1998. 